\title{
Vaccination Success and Body Condition in the European Wild Rabbit: Applications for Conservation Strategies
}

\author{
SONIA CABEZAS, ${ }^{1}$ Estación Biológica de Doñana (CSIC), 41013 Sevilla, Spain \\ CARLOS CALVETE, Instituto de Investigación en Recursos Cinegéticos (CSIC-UCLM-JCCLM), 13005 Ciudad Real, Spain \\ SACRAMENTO MORENO, Estación Biológica de Doñana (CSIC), 41013 Sevilla, Spain
}

\begin{abstract}
The European wild rabbit (Oryctolagus cuniculus) is the main prey for several endangered species and an important game species in the lberian Peninsula. However, over the last several decades 2 diseases, myxomatosis and rabbit hemorrhagic disease (RHD), have contributed to a decline in rabbit populations. In Spain, vaccination campaigns against both diseases and the translocation of vaccinated rabbits are frequently used in projects aimed at stimulating the recovery of wild populations. We estimated which factors related to body condition were associated with successful immunization by vaccination. In 191 adult wild rabbits, we calculated a body-mass index and analyzed a number of biochemical parameters and antibody concentrations before and after vaccination. Results showed that, respectively, 81 and $84 \%$ of initially seronegative rabbits seroconverted after vaccination against myxomatosis and RHD. We also found that RHD antibody concentrations after vaccination were positively associated with total protein concentrations at the moment of vaccination in all rabbits, whereas RHD antibody concentrations were negatively related to the creatinine concentration at the end of our study for initially seronegative rabbits. Our results suggested that in wild rabbits vaccination was an effective way of increasing antibody concentrations and, thus, of fighting myxomatosis and RHD, although the intensity of the immune response was conditioned by the body condition of individual rabbits. Consequently, rabbit translocations could be improved by avoiding the translocation of individuals with poor body condition and by improving vaccination protocols. In addition, success rates in mass vaccination programs carried out in free-living wild populations may be increased if the body condition of individuals is evaluated before vaccination campaigns are carried out. (JOURNAL OF WILDLIFE MANAGEMENT 70(4):1125-1131; 2006)
\end{abstract}

\section{Key words}

body condition, European wild rabbit, myxomatosis, Oryctolagus cuniculus, rabbit hemorrhagic disease, Spain, vaccination.

In the past, wildlife management was traditionally oriented towards the sustainable use of resources, with special emphasis placed on population ecology. Currently, however, strategies such as reintroductions, translocations, captive-breeding programs, habitat improvement, and the control and monitoring of diseases are essential tools in biodiversity conservation projects (Olney et al. 1994).

Given that diseases and parasites are seen to play an important role in the decline or extinction of animal populations (Viggers et al. 1993, Woodroffe 1999), infectious and noninfectious diseases are currently recognized as an ever-increasing challenge to wildlife conservation (Tompkins and Wilson 1998, Deem et al. 2001). Plans have been formulated to minimize disease impact during translocations and reintroductions (e.g., Woodford and Rossiter 1994, Cunningham 1996).

Two well-studied examples of the impact of disease on a wild species are the cases of myxomatosis and rabbit hemorrhagic disease (RHD), 2 viral diseases that affect European wild rabbits (Oryctolagus cuniculus). In the past, the native wild rabbit was a very abundant species in the Iberian Peninsula and has always been considered as the cornerstone of traditional hunting activity. Furthermore, it is the principal prey for 39 vertebrate predator species (Moreno et al. 1996). The arrival of myxomatosis in Spain in the 1950s and RHD in 1989, however, led to substantial reductions in rabbit population density (Angulo and Cooke 2002).

Today, myxomatosis and RHD are endemic diseases in the Iberian Peninsula, and periodic outbreaks occur that have a highly detrimental impact on wild rabbit populations (Calvete et al.

${ }^{1}$ E-mail:scabezas@ebd.csic.es 
2002). Rabbit declines have been especially dramatic in areas where 2 of their main predators, the Spanish Imperial Eagle (Aquila adalberti) and the Iberian Lynx (Lynx pardinus), coexist. These predators are two of the world's most threatened animals and are dependent on abundant rabbit populations (Ferrer and Negro 2004). Moreover, despite the recent history of decline in rabbit numbers, the wild rabbit is still considered the primary small-game species for sports hunting in Spain.

Consequently, over the last few years significant efforts have been made to restore and boost rabbit populations (Angulo 2003). The 2 management tools most frequently used to control diseases and improve rabbit numbers for hunting and conservationist purposes are 1) vaccination campaigns against myxomatosis and RHD carried out in wild populations (Calvete et al. 2004a,b), where rabbits are captured, vaccinated, and then immediately released in the same capture site, and 2) the restocking of populations with translocated vaccinated rabbits (Calvete et al. 1997, Calvete and Estrada 2004, Moreno et al. 2004), where wild populations are supplemented with wild rabbits (taken from other populations) that have been vaccinated against both diseases prior to their release in the new environment.

In the case of rabbit translocations, individuals are vaccinated in the hope of increasing their long-term survival. An effective immunization by vaccination against both diseases increases the long-term survival of translocated rabbits (Moreno et al. 2004), thereby increasing the possibility of success of the translocation program as the number of translocated rabbits that settle in the new environment rises (Fischer and Lindenmayer 2000). Given the high variability in antibody prevalence among rabbit donor populations and the economic and logistic costs involved in 
evaluating antibody prevalence in translocated rabbits, blind vaccination (the cheapest option) against both diseases of all translocated rabbits is performed in all translocation programs. However, immunization by vaccination does not confer permanent protection against these diseases on the young of vaccinated does (Fenner and Marshall 1954, Robinson et al. 2002). Thus, the effectiveness of both of these management tools has been questioned by hunters, conservationists, and wildlife managers in light of the generally negligible success rates.

However, despite the importance of the effective immunization against both of these diseases in the management of wild rabbit population, the evaluation of the immune response induced by vaccination with commercial vaccines has been mainly performed on standardized domestic rabbit strains in laboratory conditions. To date, only 2 studies of limited scope have been carried out on the effectiveness of vaccinating wild rabbits against RHD (Twigg et al. 1997) or against myxomatosis and RHD simultaneously (Calvete et al. 2005) and both studies provide only nonconclusive results regarding the immune response elicited by the vaccination of wild rabbits.

As part of its program aimed at conserving predator species, the Doñana National and Natural Parks (southwest Spain) have carried out wild rabbit translocations for 3 years, in which rabbits are quarantined prior to release and immunized by vaccination against myxomatosis and RHD to avoid introducing diseased animals into native rabbit populations. Our study was a part of this program and was designed to evaluate the immune response induced by vaccination against both diseases in wild rabbits. Our main objectives were 1) to estimate the levels of seroconversion in quarantined individuals after vaccination, and 2) to evaluate which factors at the moment of vaccination (biochemical parameters, body-mass, or sex) most affect the immune response to vaccines. Because the use of controlled environments can put stress on wild species and affect their immune response to vaccines, we also monitored rabbits throughout the quarantine period to evaluate the evolution of body condition during handling and restraining.

Consequently, and given the preliminary results presented by Calvete et al. (2005), we predicted that 1) the antibody concentration against both diseases will increase after vaccination, and that 2) body condition will modulate the immune response (i.e., an increase in antibody concentrations will be higher in rabbits with good body condition).

\section{Methods}

\section{Experimental Design}

From 1999 to 2002, we performed 6 translocations of adult European wild rabbits in Doñana Natural Park (SW Spain). We caught all rabbits using trap-nets in several wild populations located in Cadiz province (SW Spain). We used rabbits from this area because previous studies showed that these populations were genetically similar to Doñana wild rabbits (Branco 1995).

Immediately after capture, we transported individuals to quarantine facilities where they were kept in captivity during the study period before being released into their new environments. We sexed, weighed, and marked all rabbits with numbered metallic ear-tags, and we took their cubit lengths for the subsequent calculation of a body-mass index. For each trans- location, we randomly selected an experimental batch of rabbits (sample size ranging from 14-48 rabbits per experimental batch) with an approximately balanced sex ratio, and we took a blood sample from each of these animals to determine biochemical parameters and the initial prevalence and concentration of myxomatosis and RHD antibodies.

As the rabbits were part of the translocation program being undertaken in the Doñana Natural and National Parks, we followed their established quarantine protocols (Calvete et al. 2005). At the start of quarantine we vaccinated subcutaneously all individuals against both diseases with commercial vaccines at doses recommended for domestic rabbits (myxomatosis: live Shope fribroma virus Mixohipra-FSA, Hipra Laboratory, Gerona, Spain; RHD: ARVILAP, Ovejero Laboratory, León, Spain). During the quarantine period, we housed all animals individually in flat-decktype cages commonly used for the commercial breeding of domestic rabbits, and we provided them with food (Lucerne supplemented by $14 \%$ crude protein-commercial rabbit pellets) and water ad libitum. To avoid pathological enteric processes caused by the rapid change in diet, we progressively introduced rabbits to commercial pellets, with unlimited access 7 days after the start of quarantine.

We kept rabbits in captivity for different lengths of time $(2,3$, or 4 weeks) to conform to the release schedule planned as part of the translocation program carried out in the Doñana Natural Park. Two weeks was the minimum time the rabbits spent in quarantine, since the myxomatosis incubation period and the time following vaccination needed to develop immunity against myxomatosis and RHD in domestic rabbits, indicated that the quarantine period should last at least 12-18 days (Argüello 1986, 1991). Two days before release, we weighed all rabbits, and we obtained a second blood sample from the rabbits of the experimental batch to evaluate the change in their body condition and their seroconversion against both diseases. We took all blood samples between 1000 and 1500 hours and kept samples at room temperature until centrifugation (on the same day as they were extracted). We kept the obtained serum frozen at $-208 \mathrm{C}$ until it was analyzed in the laboratory.

For all rabbits, we calculated a body-mass index at the start and the end of the quarantine period on the basis of the residuals of a linear regression of $\log _{10}$-mass on $\log _{10}$-cubit length. This index is a measure of relative body mass corrected for differences in structural body size, and it can be estimated from ordinary leastsquares (OLS; e.g., Jakob et al. 1996, Dobson et al. 1999) or reduced major-axis regression procedures (RMA; e.g., Simmons and Scheepers 1996, Silva 1998). Given that OLS methods violate several key assumptions for calculating mass/size residual indices and may lead to type I and type II errors (Green 2001), we calculated the body-mass index by using RMA residuals. The RMA methods are considered more appropriate when $\mathrm{Y}$ and $\mathrm{X}$ are measured in different units (as in our case), and they are more robust to violations of assumptions about the ratio of the error variance (Sokal and Rohlf 1995).

\section{Biochemical and Immunological Analysis}

We determined urea nitrogen (UN), total protein (TP), and creatinine (CR) by means of spectrophotometric procedures using commercial kits (Sigma Diagnostics, Madrid, Spain). In addition, 
Table 1. Biochemical blood parameters and body-mass index according to sex, male (M) and female (F), of European wild rabbits (SW Spain), at the beginning and end of quarantine period (Nov 1999-Mar 2002). Values correspond to the mean 6 SE of all combined batches. We determined P values for the variation of mean differences between the beginning and end of quarantine period.

\begin{tabular}{|c|c|c|c|c|c|c|c|}
\hline & \multirow[b]{2}{*}{ Sex } & \multirow[b]{2}{*}{$\mathrm{n}$} & \multicolumn{2}{|c|}{ Start of captive period } & \multicolumn{2}{|c|}{ End of captive period } & \multirow[b]{2}{*}{$P$} \\
\hline & & & $\bar{x}$ & SE & $\bar{x}$ & SE & \\
\hline \multirow[t]{2}{*}{ Urea nitrogen (mg/dL) } & $\mathrm{M}$ & 87 & 28.795 & 1.024 & 26.758 & 0.838 & \\
\hline & $\mathrm{F}$ & 101 & 29.280 & 1.261 & 27.027 & 0.891 &, $0.001^{\mathrm{a}}$ \\
\hline \multirow[t]{2}{*}{ Total protein $(\mathrm{g} / \mathrm{dL})$} & M & 87 & 6.213 & 0.087 & 6.236 & 0.126 & \\
\hline & $\mathrm{F}$ & 101 & 6.563 & 0.097 & 6.718 & 0.095 & $0.003^{b}$ \\
\hline \multirow[t]{2}{*}{ Creatinine $(\mathrm{mg} / \mathrm{d})$} & $\mathrm{M}$ & 73 & 0.875 & 0.027 & 0.749 & 0.031 & \\
\hline & $\mathrm{F}$ & 80 & 0.902 & 0.063 & 0.762 & 0.061 &, $0.001^{\mathrm{a}}$ \\
\hline \multirow[t]{2}{*}{ Albumin (g/dL) } & M & 83 & 1.971 & 0.041 & 2.116 & 0.039 & \\
\hline & $\mathrm{F}$ & 93 & 2.012 & 0.040 & 2.248 & 0.040 &, $0.001^{\mathrm{a}}$ \\
\hline \multirow[t]{2}{*}{ Albumin/globulins ratio (mg/dL) } & M & 83 & 0.476 & 0.010 & 0.536 & 0.014 & \\
\hline & $\mathrm{F}$ & 93 & 0.456 & 0.011 & 0.529 & 0.014 &, $0.001^{a}$ \\
\hline \multirow[t]{2}{*}{ Body-mass index } & $\mathrm{M}$ & 92 & -0.004 & 0.006 & -0.002 & 0.006 & \\
\hline & $F$ & 106 & 0.003 & 0.007 & -0.004 & 0.006 &, $0.001^{a}$ \\
\hline
\end{tabular}

${ }^{a}$ Statistical differences were significant independent of sex.

${ }^{b}$ Statistical differences were only significant in females.

we determined concentrations of albumin $(\mathrm{AB})$ and albumin/total globulins ratio (AG) by means of electrophoresis using cellulose acetate. We used these biochemical parameters since they are indicators of tissue catabolism and nutritional status, and consequently, they are good estimators of body condition (Harder and Kirkpatrick 1994, Stirrat 2003).

We determined serum antibody concentration against myxomatosis and RHD by means of ELISAs (enzyme-linked immunosorbent assays) using commercials kits (RHD: Ingezim rabbit; Ingenasa Laboratory, Madrid, Spain; myxomatosis: CIVTest cuni; Hipra Laboratory). We expressed antibody concentration as a Relative Index of Immunity (RI), in which the optical density (OD) of each sample was related to the ODs of positive and negative controls. Relative Index of Immunity values ranged from 1-10, and we scored sera with RI Ç 2 as positive (Pages et al. 1991); that is, rabbits with RI Ç 2 had experienced effective contact with wild viral antigen prior to vaccination.

Not all biochemical and serological parameters were measured for each rabbit due to the variable amount of serum available from each individual. Thus, sample sizes were different for each of the biochemical and serological parameters analyzed.

\section{Data Analysis}

Given that handling and restraining conditions can affect the body condition of rabbits during the quarantine period (Calvete et al. 2005) and that the immune function is generally modulated by body condition (Gershwin et al. 1985, Möller et al. 1998), we first evaluated the variation in each biochemical parameter (UN, TP, $C R, A B, A G)$ and body-mass index during captivity by generalized linear mixed models for repeated measures, with 2 levels (start and end of quarantine period) for each factor, the individual as random effect, and sex (2 levels), the experimental batch (6 levels), and the length of the quarantine period (3 levels) as independent variables.

Subsequently, to evaluate the total variation in the relative immunity index (RI) experienced from the beginning to the end of the quarantine period, we performed a generalized linear mixed model for repeated measures (SAS macro program GLIMMIX, Littell et al. 1996) for each disease, with 2 levels (start and end of quarantine period) for each factor. The independent variables we used were 1) the individual as random effect, 2) sex (2 levels), 3) the experimental batch (6 levels), and 4) the length of the quarantine period (3 levels).

To test which factors (biochemical parameters, body-mass index, or sex) conditioned immune response after vaccination, we performed 4 generalized linear mixed models for each disease, with the RI at the end of quarantine period as the dependent variable, sex as independent variable and the experimental batch as the random factor. By including the batch as a random variable, we avoided pseudo-replication derived from the lack of independence in RI values from different individuals within the same experimental batch.

In the first model, our goal was to estimate the association between immune response and body condition at the moment of vaccination. Thus, we included the biochemical parameters (UN, TP, CR, AG), body-mass index, and RI at the start of quarantine period. To control for time elapsed since vaccination, we also included the length of the quarantine period (3 levels) as independent effects.

In the second model, our goal was to estimate the association between immune response and body condition at the end of quarantine. Thus, we used the same independent variables as in the first model, but the biochemical parameters and body-mass index corresponded to the end of quarantine period. The third and fourth models were performed with the same variables as the first and second models, respectively, but we used only individuals that were seronegative before vaccination, thereby excluding the individuals with previous exposure to myxomatosis and RHD antigens.

\section{Results}

Variation of Biochemical Parameters and Body-Mass During the Quarantine Period

All biochemical parameters and the body-mass index showed significant variation during our study. However, the length of the quarantine period did not affect this variation (Table 1).

Urea nitrogen and creatinine levels experienced a significant 
Table 2. Initial and final prevalence of antibodies (\%) against myxomatosis and rabbit hemorrhagic disease (RHD) and percentage of initially seronegative individuals that seroconverted after vaccination of European wild rabbits (SW Spain), in the different experimental batches (Nov 1999-Mar 2002).

\begin{tabular}{|c|c|c|c|c|c|c|}
\hline & $\begin{array}{c}\text { Batch } \\
1\end{array}$ & $\begin{array}{c}\text { Batch } \\
2\end{array}$ & $\begin{array}{c}\text { Batch } \\
3\end{array}$ & $\begin{array}{c}\text { Batch } \\
4\end{array}$ & $\begin{array}{c}\text { Batch } \\
5\end{array}$ & $\begin{array}{c}\text { Batch } \\
6\end{array}$ \\
\hline \multicolumn{7}{|l|}{ MYXOMATOSIS } \\
\hline$n$ & 18 & 14 & 46 & 22 & 40 & 35 \\
\hline Initial prevalence (\%) & 72 & 36 & 91 & 86 & 85 & 86 \\
\hline Final prevalence (\%) & 100 & 71 & 100 & 100 & 97 & 97 \\
\hline Seroconversion (\%) & 100 & 56 & 100 & 100 & 83 & 80 \\
\hline \multicolumn{7}{|l|}{ RHD } \\
\hline$n$ & 18 & 15 & 46 & 22 & 40 & 35 \\
\hline Initial prevalence (\%) & 44 & 13 & 72 & 82 & 22 & 71 \\
\hline Final prevalence (\%) & 89 & 53 & 96 & 100 & 92 & 100 \\
\hline Seroconversion (\%) & 80 & 46 & 92 & 100 & 90 & 100 \\
\hline
\end{tabular}

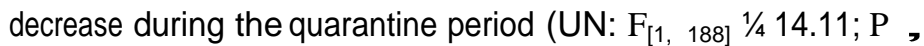
0.001; CR: $\left.\mathrm{F}_{[1,153]}\right]^{1 / 4} 37.08 ; \mathrm{P}, 0.001$ ); with a mean UN (6SE) of 29.0660 .825 and a mean CR (6SE) of 0.8960 .036 at the start of quarantine, decreasing to 26.9060 .615 and 0.7660 .035 at release, respectively. Both of these parameters also showed significant differences between experimental batches $\left(\mathrm{UN}: \mathrm{F}_{[5,188]}\right.$ $1 / 4$ 12.97; P , 0.001; CR: $\left.\left.\mathrm{F}_{[5,153]}\right]^{1 / 4} 12.97 ; \mathrm{P}, 0.001\right)$; the mean (6SE) UN ranged between 22.7061 .344 and 37.8763 .273 , and the mean (6SE) CR ranged between 0.7560 .032 and 2.12 60.813.

The total protein concentration changed in relation to sex: only in females did the total protein level increase significantly between the start and end of the quarantine period $\left(\mathrm{F}_{[1,}, 189\right] \frac{1}{1 / 4} 8.83 ; \mathrm{P} 1 / 4$ $0.003)$. We also found significant differences between experimental batches $\left(\mathrm{F}_{[5,189]}{ }^{1 / 4} 5.66 ; \mathrm{P}, 0.001\right)$; the mean $(6 \mathrm{SE}) \mathrm{TP}$ ranged between 6.0360 .082 and 6.7960 .172 .

The albumin and albumin/total globulins ratio increased significantly from the beginning to end of the quarantine period (AB: $F_{[1,175]} 1 / 426.20 ; P, 0.001 ; A G: F_{[1,175]} 1 / 432.92 ; P$, 0.001 ); with a mean $A B$ (6SE) of 1.9960 .029 and a mean AG (6SE) of 0.4760 .008 at the start of quarantine, increasing to 2.1960 .028 and 0.5360 .010 at release, respectively. These biochemical parameters also showed significant differences between experimental batches (AB: $F_{[5,175]} 1 / 46.73 ; \mathrm{P}, 0.001$; AG: $\left.F_{[5,175]} 1 / 44.33 ; P ~ 1 / 40.001\right)$; the mean (6SE) $A B$ ranged between 1.7660 .028 and 2.2060 .163 , and the mean (6SE) AG ranged between 0.4260 .010 and 0.5560 .048 .

Body-mass index values experienced a significant decline during the quarantine period $\left.\left(\mathrm{F}_{[1,198]}\right]^{1 / 4} 60.75 ; \mathrm{P}, 0.001\right)$; with a mean (6SE) of $5 \mathrm{e}^{-5} 60.004$ at the start of quarantine, decreasing to -0.00360 .004 at release, and there were differences between experimental batches $\left(\mathrm{F}_{[5,198]} 1 / 44.99 ; \mathrm{P}, 0.001\right)$; the mean (6SE) ranged between -0.0260 .009 and 0.2960 .022 .

\section{Prevalence and Seroconversion After Vaccination Against Myxomatosis and RHD}

We found rabbits with myxomatosis and RHD antibodies before vaccination in all the experimental batches in our study. The initial prevalence against the 2 diseases varied among experimental batches, ranging from $36-91 \%$ for myxomatosis, and from 1382\% for RHD (Table 2). Among rabbits that were initially seronegative against myxomatosis, the mean percentage of rabbits that seroconverted after vaccination was $81 \%$, with a range from 56-100\% according to the batch. Similarly, the mean seroconversion rate against RHD was $84 \%$, with a range from $46-100 \%$ (Table 2).

Myxomatosis antibody concentrations rose significantly after vaccination $\left.\left(\mathrm{F}_{[1,175]}\right]^{1 / 4} 162.83 ; \mathrm{P}, 0.001\right)$, with a mean relative immunity index (RI $6 \mathrm{SE}$ ) of 5.0560 .199 before vaccination increasing to 7.5460 .202 by the end of our study. There was also significant variation between experimental batches $\left(\mathrm{F}_{[5}, 175\right]{ }^{1 / 4}$ 6.22; P , 0.001; Fig 1a).

Similarly, RHD antibody concentrations significantly increased $\left(\mathrm{F}_{[1,176]}{ }^{1 / 4} 158.56 ; \mathrm{P}, 0.001\right)$, the mean RI (6 SE) increasing from 4.5060 .246 before vaccination to 7.8460 .217 by the end of survey. Significant differences were also found between experimental batches $\left(\mathrm{F}_{[5,176]} 1 / 413.49 ; \mathrm{P}, 0.001\right.$; Fig 1b). However, for neither of the 2 diseases did the mean RI show significant differences for sex or the length of quarantine period.

Factors Affecting the Myxomatosis Immune Response Myxomatosis antibody concentrations by the end of the quarantine period were positively related to the initial RI value $\left(F_{[1,168]}{ }^{1 / 4} 72.97 ; P, 0.001\right)$. However, none of the variables at the start or the end of the quarantine period showed any significant association with antibody concentrations. When analyzing only the initially seronegative individuals, none of the variables corresponding to the start or to the end of the study had a significant effect on the final concentration of antibodies. Finally, none of the models detected any significant association between antibody concentrations and sex or the length of the quarantine period.

\section{Factors Affecting the RHD Immune Response}

The antibody concentration after vaccination against RHD was positively correlated to the initial level of total proteins $\left(\mathrm{F}_{[1,158]}\right]^{1 / 4}$ 4.32; $\mathrm{P} 1 / 40.039)$ and to the initial $\mathrm{RI}$ value $\left(\mathrm{F}_{[1,158]}\right]^{1 / 4} 8.75 ; \mathrm{P} 1 / 4$ 0.004). However, none of the variables at the end of quarantine period were associated with the final RI value.

For the initially seronegative individuals, the albumin/total globulins ratio before vaccination was inversely related to the final antibody concentration $\left(\mathrm{F}_{[1,} 64\right]^{1 / 4} 6.34 ; \mathrm{P}^{1 / 4}$ 0.014). Furthermore, the creatinine level obtained at the end of the quarantine period was negatively associated with the final $R I$ value $\left(F_{[1,61]} 1 / 44.21 ; P\right.$ $1 / 40.045$ ). For none of the analyzed models was the intensity of immune response related to sex or to length of the quarantine period.

\section{Discussion}

Our results suggested that, although immunization against myxomatosis and RHD by vaccination was modulated by body condition, it may be an effective prophylactic tool in wild rabbits at the individual level. Antibody concentrations significantly increased after vaccination, despite restraining rabbits in a new and probably stressful environment, with the likelihood of affecting their immune response (especially among the initially seronegative rabbits).

Evaluating the effect of vaccinating wild animals is difficult, particularly given the number of individuals previously exposed to 
wild antigens and the resulting variability in prevalence of natural antibodies. We found rabbits with antibodies for both diseases in all sampled populations, which resulted in an important variation in antibody prevalence in the experimental batches. This variability in antibody prevalence agreed with results found in other natural wild rabbit populations and reflected variations in transmission rates or in the immunological status of different populations (Trout 1997, Marchandeau et al. 2000).

In addition, handling and restraining can place stress on wild individuals affecting body condition and therefore their immune response to vaccines. We found decreased urea nitrogen and creatinine levels and increased albumin and albumin/total globulins ratio, as well as a gender-specific increase in total protein concentrations. Tissue catabolism as a response to stressful stimuli can increase creatinine and urea nitrogen blood concentrations (Jacobson et al. 1978, Amici et al. 2000). Blood urea nitrogen is also one of the most widely used indices of nutritional status (Harder and Kirkpatrick 1994) since it is inversely correlated to the quality and quantity of protein intake (Bora et al. 2001). In addition, the quantity of protein consumed can also be positively associated with total serum proteins and albumin levels (Warren and Kirkpatrick 1978).

Therefore, and in agreement with the findings of Calvete et al. (2005) derived from a study of wild rabbits handled and restrained in similar conditions to our study, the changes in the biochemical parameters recorded throughout the captive period may be due to a decrease in the acute stress experienced after initial capture and handling or to the change in nutritional status caused by the intake of high-quality proteins included in the diet. However, and just as Calvete et al. (2005) concluded, the lack of baseline reference values for rabbit biochemical parameters in the wild precluded us from evaluating the degree of adaptation of these rabbits to restraint during captivity.

The body-mass index was lower at the end than at the start of the quarantine period, probably as consequence of the loss of mass caused by the limited access to high-quality food (commercial pellets) during the first days of restraint. Under the same quarantine protocols, Calvete et al. (2005) showed that rabbits need several weeks of unlimited access to high-quality food to recover body-mass values similar to those at the start of quarantine.

In accordance with our predictions, our results showed that vaccination significantly increased antibody prevalence and concentrations against both diseases. However, in contrast to experimental surveys carried out on domestic rabbit strains (Argüello 1991, Marlier et al. 2000), the seroconversion of initially seronegative individuals was not $100 \%$ effective, but it ranged from between $56-100 \%$ for myxomatosis and between $46-$ $100 \%$ for RHD. At the individual level, this variability was probably due to the variety found in body condition among wild individuals; nevertheless, differences observed between experimental batches in seroconversion rates were difficult to explain. Calvete et al. (2005) also obtained low seroconversion mean values in initially seronegative rabbits $(69 \%$ and $45 \%$ for myxomatosis and RHD, respectively). These figures contrasted with the high percentage of seroconversion estimatedfor the remaining experimental batches in our study, suggesting that some factors (a)

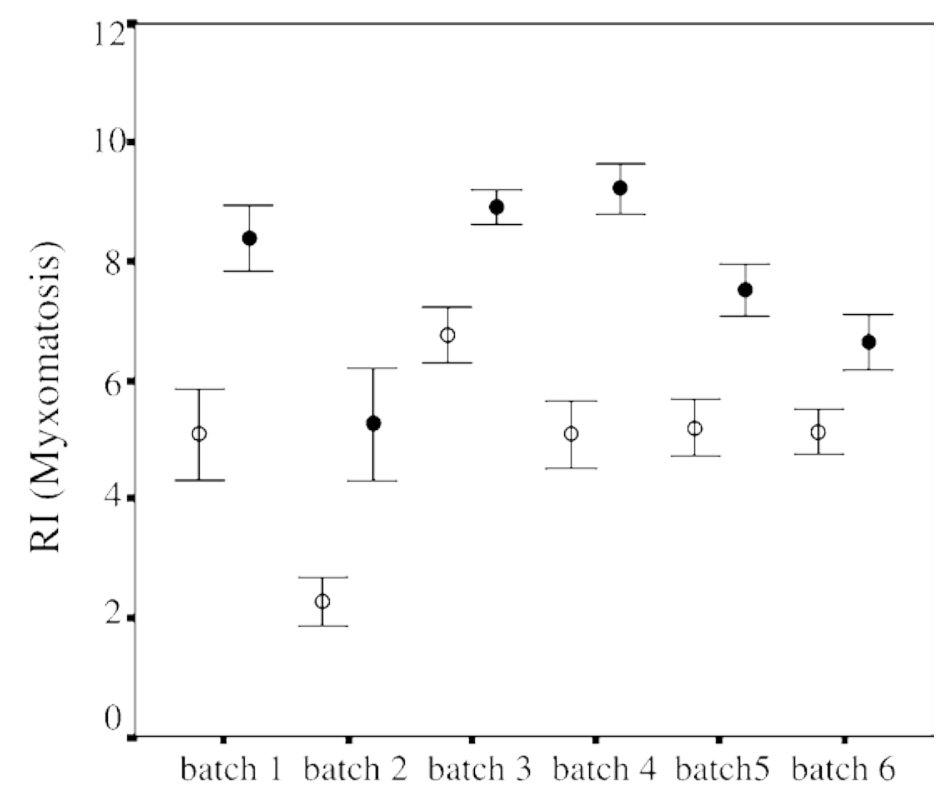

(b)

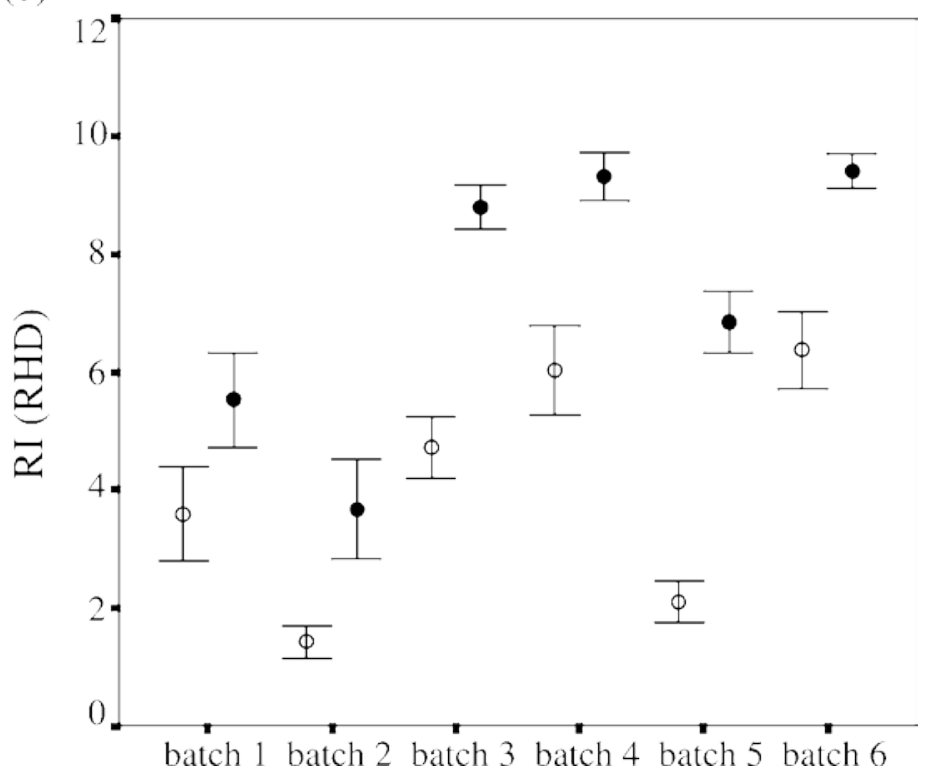

Figure 1. Average 6 SE relative index of immunity (RI) value in myxomatosis (a) and rabbit hemorrhagic disease (RHD; b) of European wild rabbits (SW Spain), before (white) and after (black) vaccination against these diseases in the 6 experimental batches (Nov 1999-Mar 2002). In all experimental batches, for both diseases, the variation in the average RI value between the beginning and end of quarantine period showed a $P$ value, 0.001

affecting rabbits at batch level or affecting the effectiveness of vaccines (e.g., a failure in storage conditions in the consignment of vaccines somewhere between the laboratory and the quarantine facilities) were not controlled.

We found that some biochemical blood parameters were closely linked to the magnitude of the immune response. The antibody concentration induced by vaccination against RHD was positively associated with the initial concentration of total proteins for all individuals. Our results suggested that rabbits with better body condition at the moment of vaccination experienced greater 
immune response, given that the total serum protein level is considered a good direct nutritional indicator and estimate of body condition (Harder and Kirkpatrick 1994). This result agrees with our predictions and is supported by studies of other mammalian and bird taxa, which have demonstrated that the immune function is generally positively correlated to body condition (e.g., Gershwin et al. 1985, Möller et al. 1998).

Creatinine can be used as an indicator of muscle damage, and elevated levels are associated with reduced food intake, mass loss, and decreases in body fat and protein (DelGiudice et al. 1990). In our study, rabbits that were seronegative prior to vaccination showed a negative association between their final creatinine level and the concentration of antibodies against RHD after vaccination, suggesting that the immune response of rabbits without past exposure to the RHD antigen was modulated by the change in their body condition during our study. These rabbits also showed a negative association between the initial albumin/total globulins ratio and the antibody concentration acquired after vaccination against RHD. The physiological mechanisms involved herein are difficult to explain. One possibility is that, given that the gamma-fraction of globulins comprises mainly antibodies, those rabbits with high globulin levels (mainly due to high concentrations of gamma-globulins) and, therefore, a low albumin/total globulins ratio, were in fact animals with low RHD antibody concentrations that had prior contact with the RHD antigen. This low antibody concentration precluded that we classify them as seropositives by means of the ELISA test. This way, vaccination merely had a booster effect on the production of RHD antibodies, just as in the remaining initially seropositive rabbits, and there was no need for them to develop the proper immunologic mechanisms against a novel antigen.

In the case of myxomatosis, no biochemical factor was found to be associated with the immune response induced by vaccination against this disease, probably due to the higher initial prevalence of natural myxomatosis antibodies in comparison with RHD antibodies. This difference in antibody prevalence between these diseases has already been described in wild rabbit populations (Calvete et al. 2002).

\section{Literature Cited}

Amici, A., O. France, P. Mastrodiacono, N. Merendino, M. Nardini, and G. Tomassi. 2000. Short-term acute heat stress in rabbits: functional, metabolic and immunological effects. World Rabbit Science 3:11-16.

Angulo, E. 2003. Factores que afectan a la distribución y abundancia del conejo en Andalucía. Dissertation, Complutense University, Madrid, Spain. [In Spanish.]

Angulo, E., and B. D. Cooke. 2002. First synthesize new viruses then regulate their release? The case of the wild rabbit. Molecular Ecology 11:2703-2709.

Argüello, J. L. 1986. Contribución a la profilaxis de la mixomatosis del conejo mediante el uso de una cepa homóloga. Medicina Veterinaria 3:91-103. [In Spanish.]

Argüello, J. L. 1991. La enfermedad hemorrágica viral del conejo: vacunación y repuesta inmune. Revue Scientifique et Technique Office International des Epizooties 10:459-470. [In Spanish.]

Bora, A., R. N. Baruah, K. K. Baruah, and R. K. Bora. 2001. Influence of different concentrate: roughage ratio on certain blood constitute of rabbits. Indian Veterinary Journal 78:1044-1045.

Branco, M. 1995. Contribuçao para o estudo da genética bioquímica e populacional do coelho, Oryctolagus cuniculus. Dissertation, Porto University, Porto, Portugal. [In Portuguese.]

Calvete, C., E. Angulo, R. Estrada, S. Moreno, and R. Villafuerte. 2005.
Finally, we found that the antibody concentration induced by vaccination against these 2 diseases was not associated with the number of days elapsed since vaccination. Previous studies have shown that antibodies against these viruses attain stable levels during the first 2 weeks after vaccination (Argüello 1991). Given that in our study the duration of the captive period was equal to or longer than 2 weeks, we did not find any association between antibody concentration and the duration of the captive period.

\section{Management Implications}

If vaccination is carried out in restrained wild animals, we recommend that the immune response after vaccination be evaluated to establish practices for improving immunization techniques. For example, a booster vaccination can be used at the end of captive period (after testing to see if body condition has improved) to increase antibody concentration and, therefore, longterm immunization. However, in mass immunization programs carried out in free-living wild populations, where the establishment of prior practices for improving immunization is impracticable, the success of vaccination will be conditioned by the body condition of individuals at the moment of vaccination. Therefore, we recommend that the monitoring and evaluation of the body condition of individuals that compose the populations be performed previouslyas a means of optimizing vaccination programs.

\section{Acknowledgments}

We thank A. Melero, L. Lombardi, and P. Rubio for their help during data collection, and Drs. J. Blas, E. Angulo, and M. Delibes for their useful comments and for revising previous drafts of the manuscript. Dr. R. Villafuerte kindly allowed us to perform the laboratory analyses at the Instituto de Investigación en Recursos Cinegéticos. The staff of Doñana Natural Park provided technical and logistical support. We also thank Drs. M. L. Morrison and C. Chambers, and 2 anonymous reviewers for their comments, which significantly improved the manuscript. The research was supported by FEDER (1FD1997-0789) and MCYT (BOS2001-2391-C02-01) projects.

Quarantine length and survival of translocated European wild rabbits. Journal of Wildlife Management 69:1063-1072.

Calvete, C., and R. Estrada. 2004. Short-term survival and dispersal of translocated European wild rabbits. Improving the release protocol. Biological Conservation 120:507-516.

Calvete, C., R. Estrada, J. Lucientes, J. J. Osacar, and R. Villafuerte. 2004a. Effects of vaccination against viral haemorrhagic disease (VHD) and myxomatosis on long-term mortality rates of European wild rabbits. The Veterinary Record 155:388-392.

Calvete, C., R. Estrada, J. J. Osacar, J. Lucientes, and R. Villafuerte. 2004b. Short-term negative effects of vaccination campaigns against Myxomatosis and viral haemorrhagic disease (VHD) on the survival of European wild rabbits. Journal of Wildlife Management 68:198-205.

Calvete, C., R. Estrada, R. Villafuerte, J. J. Osácar, and J. Lucientes. 2002. Epidemiology of viral haemorrhagic disease and Myxomatosis in free-living population of wild rabbits. Veterinary Record 150:776-782.

Calvete, C., R. Villafuerte, J. Lucientes, and J. J. Osácar. 1997. Effectiveness of traditional wild rabbit restocking in Spain. Journal of Zoology, London 241: $1-7$.

Cunningham, A. A. 1996. Disease risk of wildlife translocations. Conservation Biology 10:349-353. 
Deem, S. L., W. B. Karesh, and W. Weisman. 2001. Putting theory into practice: wildlife health in conservation. Conservation Biology 15:12241233.

DelGiudice, G. D., L. D. Mech, and U. S. Seal. 1990. Effects of winter undernutrition on body composition and physiological profiles of white-tailed deer. Journal of Wildlife Management 54:539-550.

Dobson, F. S., T. S. Risch, and J. O. Murie. 1999. Increasing returns in the life history of Columbian ground squirrels. Journal of Animal Ecology 68:73-86.

Fenner, F., and I. D. Marshall. 1954. Passive immunity in Myxomatosis of the European rabbit: the protection conferred on kittens born by immune does. Journal of Hygiene 52:321-336.

Ferrer, M., and J. J. Negro. 2004. The near-extinction of two large European predators: the super-specialists pay a price. Conservation Biology 18:344349.

Fischer, J., and D. B. Lindenmayer. 2000. An assessment of the published results of animal relocations. Biological Conservation 96:1-11.

Gershwin, M. E., R. S. Beach, and L. S. Hurley. 1985. Nutrition and immunity. Academic, New York, New York, USA.

Green, A. J. 2001. Mass/length residuals: measures of body condition or generators of spurious results? Ecology 82:1473-1483.

Harder, D. J., and L. R. Kirkpatrick. 1994. Physiological methods in wildlife research. Pages 275-306 in T. A. Bookhout, editor. Research and management techniques for wildlife and habitats. The Wildlife Society, Bethesda, Maryland, USA.

Jacobson, H. A., R. L. Kirkpatrick, and B. S. Mcginnes. 1978. Disease and physiologic characteristics of two cottontail populations in Virginia. Wildlife Monographs 60:5-53.

Jakob, E. M., S. D. Marshall, and G. W. Uetz. 1996. Estimating fitness: a comparison of body condition indices. Oikos 77:61-67.

Littell, R. C., G. A. Milliken, W. W. Stroup, and R. D. Wolfinger. 1996. SAS System for mixed models. SAS Institute, Cary, North Carolina, USA.

Marchandeau, S., Y. Chaval, and E. Le Goff. 2000. Prolonged decline in the abundance of wild European rabbits Oryctolagus cuniculus and high immunity level over three years following the arrival of rabbit haemorrhagic disease. Wildlife Biology 6:141-147.

Marlier, D., J. Mainil, C. Boucraut, A. Linden, and H. Vindevogel. 2000. The efficacy of two vaccination schemes against experimental infection with a virulent amyxomatous or a virulent nodular myxoma virus strain. Journal of Comparative Pathology 122:115-122.

Möller, A. P., P. Christe, J. Erritzoe, and J. Mavarez. 1998. Condition, disease and immune defense. Oikos 83:301-306.

Moreno, S., R. Villafuerte, S. Cabezas, and L. Lombardi. 2004. Wild rabbit restocking for predator conservation in Spain. Biological Conservation 118: 183-193.
Moreno, S., R. Villafuerte, and M. Delibes. 1996. Cover is safe during the day but dangerous at night: the use of vegetation by European wild rabbits. Canadian Journal of Zoology 74:1656-1660.

Olney, P. J. S., G. M. Mace, and A. T. C. Feistner, editors. 1994. Creative conservation: interactive management of wild and captive animals. Chapman and Hall, London, United Kingdom.

Pages, A., C. Artigas, and E. Espuña. 1991. Serological profile (by ELISA) of the active and passive immunity on rearing does vaccinated with and oil inactivated vaccine against RHD. Proceedings of International Symposium on RHD. 6-10 August 1991, Beijing, China.

Robinson, A. J., P. T. M. So, W. J. Muller, B. D. Cooke, and L. Capucci. 2002. Statistical models for the effect of age and maternal antibodies on the development of rabbit haemorrhagic disease in Australian wild rabbits. Wildlife Research 29:663-671.

Silva, M. 1998. Allometric scaling of body length: elastic or geometric similarity in mammalian design. Journal of Mammalogy 79:20-32.

Simmons, R. E., and L. Scheepers. 1996. Winning by a neck: sexual selection in the evolution of giraffe. American Naturalist 148:771-786.

Sokal, R. R., and F. J. Rohlf. 1995. Biometry. Third edition. W. H. Freeman, New York, New York, USA.

Stirrat, S. C. 2003. Body condition and blood chemistry of agile wallabies (Macropus agilis) in the wet-dry tropics. Wildlife Research 30:59-67.

Tompkins, D. M., and K. Wilson. 1998. Wildlife disease ecology: from theory to policy. Trends in Ecology and Evolution 13:476-478.

Trout, R. C. 1997. Seroepidemiology of rabbit haemorrhagic disease (RHD) in wild rabbits (Oryctolagus cuniculus) in the United Kingdom. Journal Zoological London 243:846-853.

Twigg, L. E., A. G. Wheeler, and J. Parkinson. 1997. Adverse reactions in wild, free-ranging European rabbits vaccinated against rabbit haemorrhagic virus. Australian Veterinary Journal 75:448-449.

Viggers, K. L., D. B. Lindenmayer, and D. M. Spratt. 1993. The importance of disease in reintroduction programmes. Wildlife Research 20:687-698.

Warren, R. J., and R. L. Kirkpatrick. 1978. Indices of nutritional status in cottontail rabbits fed controlled diets. Journal of Wildlife Management 42: 154-158.

Woodford, M. H., and P. B. Rossiter. 1994. Disease risks associated with wildlife translocation projects. Pages 178-200 in P. J. S. Olney, G. M. Mace, and A. T. C. Feistner, editors. Creative conservation: interactive management of wild and captive animals. Chapman and Hall, London, United Kingdom.

Woodroffe, R. 1999. Managing disease threats to wild mammals. Animal Conservation 2:185-193.

Associate Editor: Chambers. 